\title{
Genome-wide analyses of metal responsive genes in Caenorhabditis elegans
}

\section{Samuel Caito ${ }^{1,2}$, Stephanie Fretham ${ }^{1}$, Ebany Martinez-Finley 1,2, Sudipta Chakraborty 2,3, Daiana Avila ${ }^{4}$ Pan Chen ${ }^{1}$ and Michael Aschner ${ }^{1,2,3,5}$ *}

\author{
${ }^{1}$ Division of Clinical Pharmacology and Pediatric Toxicology, Department of Pediatrics, Vanderbilt University Medical Center, Nashville, TN, USA \\ ${ }^{2}$ Center in Molecular Toxicology, Vanderbilt University Medical Center, Nashville, TN, USA \\ ${ }^{3}$ Center for Molecular Neuroscience, Vanderbilt University Medical Center, Nashville, TN, USA \\ ${ }^{4}$ Biochemistry Graduate Program, Universidade Federal do Pampa, Uruguaiana, Rio Grande do Sul, Brazil \\ ${ }^{5}$ The Kennedy Center for Research on Human Development, Vanderbilt University Medical Center, Nashville, TN, USA
}

Edited by:

Stephen Sturzenbaum, King's College London, UK

\section{Reviewed by:}

Jonathan Freedman, National Institute of Health, USA

Suresh Chandra Swain, Aberystwyth University, UK

\section{*Correspondence:}

Michael Aschner, Division of Pediatric Toxicology, Vanderbilt University Medical Center, 11425 MRB IV, 2215-B Garland Avenue, Nashville, TN 37232-0414, USA.

e-mail:michael.aschner@

vanderbilt.edu
Metals are major contaminants that influence human health. Many metals have physiologic roles, but excessive levels can be harmful. Advances in technology have made toxicogenomic analyses possible to characterize the effects of metal exposure on the entire genome. Much of what is known about cellular responses to metals has come from mammalian systems; however the use of non-mammalian species is gaining wider attention. Caenorhabditis elegans is a small round worm whose genome has been fully sequenced and its development from egg to adult is well characterized. It is an attractive model for high throughput screens due to its short lifespan, ease of genetic mutability, low cost, and high homology with humans. Research performed in C. elegans has led to insights in apoptosis, gene expression, and neurodegeneration, all of which can be altered by metal exposure. Additionally, by using worms one can potentially study mechanisms that underline differential responses to metals in nematodes and humans, allowing for identification of novel pathways and therapeutic targets. In this review, toxicogenomic studies performed in $C$. elegans exposed to various metals will be discussed, highlighting how this non-mammalian system can be utilized to study cellular processes and pathways induced by metals. Recent work focusing on neurodegeneration in Parkinson's disease will be discussed as an example of the usefulness of genetic screens in $C$. elegans and the novel findings that can be produced.

Keywords: metals, gene expression, C. elegans, apoptosis, neurodegeneration

\section{INTRODUCTION}

Metals are persistent environmental contaminants that have been associated with developmental and behavioral deficits and neurodegeneration (Kordas, 2010; Koyashiki et al., 2010; Neal and Guilarte, 2010). Several metals, such as $\mathrm{Zn}, \mathrm{Mn}$, and Fe, are essential for cell viability and function; however excessive levels of metals can be deleterious. Metals can generate free radicals and reactive oxygen species (ROS) through Fenton chemistry, leading to protein and DNA oxidation and lipid peroxidation. Oxidation of macromolecules can damage cellular organelles and generate additional ROS, thus creating a vicious cycle. Exposure to metals can range in their clinical symptoms, but similar cellular effects are observed, such as oxidative stress. With the advent of complete genome sequencing and genome-wide mapping, it is now

Abbreviations: AD, Alzheimer's disease; AO, acridine orange; C. elegans, Caenorhabditis elegans; DAergic, dopaminergic; DTT, dichlorodiphenylchloroethane; eIF $2 \alpha$, eukaryotic translation initiation factor; ER, endoplasmic reticulum; ERAD, ERassociated degradation; HD, Huntington's disease; HSP, heat shock protein; LRRK2, leucine-rich repeat kinase 2; Nrf2, nuclear factor (erythoid-derived 2)-like 2; PD, Parkinson's disease; PINK1, PTEN-induced novel kinase 1; PTEN, phosphatase and tensin homolog; $\mathrm{SNpc}$, substantia nigra pars compacta; $\mathrm{ROS}$, reactive oxygen species; UPR, unfolded protein response. possible to identify novel genes involved in metal homeostasis, detoxification, and involved in response to toxic exposures. One important consideration in performing a toxicogenomic screen is the choice of model organism. Traditional mammalian models, such as rats and mice have provided vital information about metal exposures. However rodents are complex organisms where subtle, yet important, changes may not be observed due to the numerous cell types and their relative abundance in the tissue examined. Non-mammalian model organisms provide an alternative, less complex system to perform these screens while maintaining high homology to mammals.

Caenorhabditis elegans is an attractive model for studying effects of metals on gene expression. C. elegans are small $(\sim 1.5 \mathrm{~mm})$ round worms with a short lifespan of 3 weeks and a life cycle of 3 days, that can be maintained cheaply in a humid environment containing atmospheric oxygen and bacteria grown on agar as a food source. A single adult hermaphrodite is capable of producing 300 progeny. Upon hatching (L1 phase), worms proceed through three subsequent larval stages (L2 after $12 \mathrm{~h}, \mathrm{~L} 3$ after $8 \mathrm{~h}$, and $\mathrm{L} 4$ after $10 \mathrm{~h}$ ). As an adult, an hermaphrodite is able to self-fertilize, or may be used for mating with an adult male, a rare sex with a frequency of $0.5 \%$, allowing for crossing 
worms of different genotypes. Importantly, worms contain many of the transporters and stress response genes critical for xenobiotic and metal detoxification, including metallothioneins (MTs), transporters involved in metal homeostasis, heat shock proteins (HSPs), and genes involved in glutathione homeostasis.

Because of the short lifespan and ease of manipulability, $C$. elegans are a perfect model for high throughput toxicogenomic screens. C. elegans' body comprises less than 1000 cells, allowing for the mapping of every cell's development and lineage (Sulston and Horvitz, 1977). This allows one to investigate not only general overall toxicity, but neurotoxicity, reproductive toxicity, muscular toxicity, toxicity to the GI track, and developmental toxicity. The C. elegans genome is fully sequenced and shows 60$80 \%$ homology with mammals (Kaletta and Hengartner, 2006; McDonald et al., 2006). The genetic architecture of C. elegans genes is well characterized and there are standardized protocols for knocking down genes through RNAi and introducing DNA through injections, allowing for the creation of numerous strains of knockout alleles and transgenic overexpression alleles. These advantages allow for quick but informative investigations in signaling pathways and gene-environment interactions that may be more difficult in more complex systems. It is possible that genes induced by metal exposure in C. elegans may differ from mammals, but this may provide insight into pathways and protective genes that can be investigated as therapeutic targets. In this review, we highlight recent toxicogenomic screens in $C$. elegans, including screens in various worm lines, RNAi feeding screens, and microarray analyses, to characterize genes and pathways altered by metal exposure. Additionally we discuss novel findings from studies in C. elegans of metal-induced neurodegeneration in Parkinson's disease (PD) as an application of toxicogenomic screens in studying human disease in a non-mammalian system.

\section{DNA DAMAGE, GENE EXPRESSION, EPIGENETICS}

Various metals damage DNA, change gene expression profiles, and epigenetically modify DNA, consequences which can contribute to the toxicity of metal exposure. Many of these alterations have been identified and characterized using mammalian systems (Bal and Kasprzak, 2002; Beyersmann and Hartwig, 2008; Salnikow and Zhitkovich, 2008; Robinson et al., 2010), however there are novel genetic screens in C. elegans that can be utilized to examine metal-induced genetic changes.

A survey for naturally occurring single base substitutions assayed the entire genomes of 10 , mutation accumulation $C$. elegans strains generated by bottlenecking individual wildtype animals for many generations (Denver et al., 2009). High throughput synthesis- and pyro-DNA sequencing revealed more base substitutions than previous studies and found that $\mathrm{G}: \mathrm{C} \rightarrow \mathrm{A}: \mathrm{T}$ and $\mathrm{G}: \mathrm{C} \rightarrow \mathrm{T}: \mathrm{A}$ mutations occurred at higher than expected rates, indicative of oxidative DNA damage. While this study did not directly examine metal exposure, it suggests that, as in mammalian systems, oxidative stress resulting from metals likely causes DNA damage in C. elegans (Bal and Kasprzak, 2002). DNA strand breaks are another form of DNA damage induced by exposure to metals such as $\mathrm{Cd}, \mathrm{Cu}$, and $\mathrm{Zn}$ in C. kiiensis invertebrate larvae (Al-Shami et al., 2012). This single cell assay has not been applied specifically to metal toxicity in C. elegans, however it has been used to examine nicotine genotoxicity (Sobkowiak and Lesicki, 2009).

Microarray-technology alone and in combination with other techniques, has demonstrated changes in gene expression following $\mathrm{Ag}$ and $\mathrm{Cd}$ exposure C. elegans. Whole genome microarray identified altered expression of 1632 genes after exposure to $\mathrm{Ag}$ nanoparticles and ions, including genes involved in oxidative stress and metabolism (Roh et al., 2009). In addition, this study combined genetic changes with in vivo toxicological outcomes of survival, growth, and reproduction. Another study identified altered expression of 290 genes using whole genome microarray following Cd exposure (Cui et al., 2007). Many of the Cd-altered genes had previously known functions including metabolism, proteolysis, fatty acid, and lipid metabolism, although the majority of identified genes were novel. RNAi against 92 of the most significantly altered genes was used to screen for interactions between gene function and growth and reproductive phenotypes associated with Cd exposure (Cui et al., 2007).

The short lifespan of C. elegans, presence of epigenetic machinery, and heritability of epigenetic alterations (Greer et al., 2011; Wenzel et al., 2011) make it possible to examine epigenetic contributions to metal toxicity in the worm. One recent study combined several genomic assays including microarray, ChIP-seq with histone modification specific antibodies, and RT-PCR to assess global genomic differences between adults that had experienced Dauer and those that had not (Hall et al., 2010). Such an approach has not yet been applied to the toxicogenomic consequences of metal exposure, but would be highly informative particularly for non-lethal and latent toxicity.

\section{ANTIOXIDANT RESPONSE TO METAL EXPOSURE}

Another way in which metals damage cells is through oxidative stress. There are several C. elegans mutants that are hypersensitive to oxidative stress including mev-1, mev-3, gas-1, rad-8, skn-1, and nnt-1 (Ishii et al., 1990; Yamamoto et al., 1996; Hartman et al., 2001; Kayser et al., 2001; An and Blackwell, 2003; Arkblad et al., 2005), with $n n t-1$, and $s k n-1$ primarily involved in antioxidant defense. Many genes are involved in generation of ROS, Fujii et al. (2009) screened for $C$. elegans mutants that showed increased sensitivity to oxidative stress, and isolated a novel mutant, oxy-4(qa5001) (Fujii et al., 2009). This mutant showed an increased sensitivity to a high concentration of oxygen, and decreased longevity that is temperature-dependent. Genetic analysis showed that $o x y-4$ had a mutation in an [FeFe]-hydrogenase-like gene, responsible for catalyzing the formation and the splitting of molecular hydrogen $\left(\mathrm{H}_{2} \leftrightarrow 2 \mathrm{H}^{+}+2 \mathrm{e}^{-}\right)$, and functioning in anaerobic respiration but whose properties are less characterized in aerobic eukaryotes. Results from this study suggest that [FeFe]-hydrogenase-like genes are involved in the regulation of sensitivity to oxygen in C. elegans (Fujii et al., 2009).

The daf-2 insulin-like signaling pathway plays a major role in $C$. elegans longevity by controlling the expression of a large number of genes, including free radical detoxifying genes (Honda and Honda, 1999). Samuelson and colleagues performed a genome-wide RNAi screen to identify genes necessary for the extended lifespan of daf2 mutants and identified $\sim 159$ gene inactivations responsible for shortening the lifespan. Endocytosis and vesicular trafficking to 
lysosomes made up a majority of the genes identified for lifespan extension in the daf-2 mutant. Decreased daf-2 signaling causes nuclear localization of DAF-16 and results in upregulation of manganese superoxide dismutase (SOD-3), whose function is to protect cells from oxidative stress. Thirty-four gene inactivations were identified that suppressed the induction of SOD-3 expression in non-neuronal cells (Samuelson et al., 2007).

Toxicogenomic studies have described the utility of C. elegans as an environmental monitor and biosensor. Chu et al. (2005) tested a number of mutant strains defective in genes controlling ROS response for enhanced sensitivity to metals. A double mutant daf-16 unc-75 strain was identified as most sensitive, exhibiting a sixfold increase in sensitivity to Cd, threefold increase in sensitivity to $\mathrm{Cu}$, and twofold increase in sensitivity to $\mathrm{Zn}$ compared to wildtype worms. Roh et al. (2006) investigated the toxicity of $\mathrm{Cd}, \mathrm{Pb}$, $\mathrm{Cr}$, and As using a transgenic C. elegans model. Cd led to a more than threefold increase in HSP 16.2, HSP 70, metallothionein 2, cytochrome P450 family protein 35A2, glutathione-S-transferase 4, superoxide dismutase 1 , catalase 2, C. elegans p53-like protein 1 , and apoptosis enhancer 1 genes compared to controls. The $\mathrm{Pb}$ , Cr-, and As-exposed nematodes showed little change in gene expression showing that $\mathrm{Cd}$ has a higher tolerance level compared to the other metals tested. Cd- and Cr-exposed worms also exhibited alterations in growth and reproduction. This study illustrated that stress responses must be measured following exposure to several different metals (Roh et al., 2006). The DNA microarray experiments of Cui et al. (2007) examined the toxicogenomic response to $\mathrm{Cd}$ and identified 237 up-regulated and 53 downregulated genes that significantly changed following either 4 or $24 \mathrm{~h}$ exposure. Early response genes were those that regulate the localization and transport of various metals and are important for ion homeostasis. Cd exposure resulted in the overexpression of 25 biotransformation genes, proteolysis genes, and expression of four ABC transporters, $p g p-1, p g p-8, p g p-9$, and $m r p-3$. Fatty acid and cellular lipid metabolism and cell wall catabolism pathways represented a significant portion of the downregulated gene set (Cui et al., 2007).

Caenorhabditis elegans' SKN-1 [mammalian nuclear factor (erythoid-derived 2)-like 2, Nrf2] is critical for oxidative stress resistance. Wang et al. (2009) conducted a genome-scale RNAi screen to identify mechanisms that prevent inappropriate $s k n-1$ target gene expression under non-stressed conditions (Wang et al., 2009). They identified 41 genes that if knocked down lead to activation of a SKN-1 target gene, gcs-1. These genes represent many cellular processes, including mRNA translation. Results suggest that SKN-1 acts as a monitor of many metabolic and regulatory processes.

\section{HEAT SHOCK PROTEINS AND ER STRESS}

Cellular functions are greatly dependent upon efficient and accurate cooperation between different proteins, relying on efficient protein synthesis, processing, trafficking, and degradation. Thus, it is not surprising that cells have evolved several defense mechanisms to address misfolded and damaged proteins. Molecular chaperone proteins are highly conserved and ubiquitously expressed in all subcellular compartments and are essential for the stability of the proteome under normal and stressful conditions
(Frydman, 2001). The expression of many molecular chaperones is regulated by different forms of environmental and physiological stresses that can interfere with folding stability, thus leading to a flux of misfolded proteins. Stress responsive molecular chaperones are referred to as HSPs and classified by gene families according to their molecular mass. HSPs exert their physiological effect by assisting in the formation of new proteins as well as by preserving existing structures. However, they also display major functions in pathological conditions, especially through structural rectification of denatured proteins and solubilization of protein aggregates carrying them on to the proteasome system (Soti et al., 2005; Powers et al., 2010).

Despite the robust nature of the heat shock response and the capacity of chaperones to recognize misfolded proteins, chronic exposure to metals even at low doses, or acute toxicity at high doses may result in the accumulation of misfolded and damaged proteins. The accumulation of alternate folded states and toxic species overburdens and functionally depletes the proteostasis machinery, which in turn amplifies protein damage (Gidalevitz et al., 2006, 2009). This suggests that the regulation of the protein quality control system is essential for proteostasis to monitor the state of the proteome throughout the lifetime of an organism. Hence, HSP expression has emerged as an indicator of cellular stress in animals exposed to metals at sub-toxic and toxic doses.

Toxicogenomics has helped in elucidating whether HSP levels are altered by metals exposure. HSP levels vary after metal exposure depending on the metal, type of cell or organ studied, and type of chaperone evaluated (Cheng et al., 2003; Qian et al., 2005; Rodella et al., 2008; Escobar Mdel et al., 2009). Some metals such as $\mathrm{Pb}, \mathrm{Cd}, \mathrm{Al}$, and $\mathrm{Hg}$ can directly inhibit protein folding or inhibit their expression, thus causing accumulation of unfolded proteins and endoplasmic reticulum (ER) stress (Rodella et al., 2008; Sharma et al., 2008; Aremu et al., 2011). There are several diseases associated with long-term metal exposure, increased misfolded protein aggregation, and disturbance in the ubiquitin/proteasome system including Alzheimer's disease (AD), PD, and polyglutamine diseases, such as Huntington's disease (HD; Imaizumi et al., 2001; Gitler et al., 2009; Squitti, 2012). Once proteins are misfolded, they can aggregate and damage the cell. Metal-induced oxidative stress has been shown to promote protein aggregation (Santner and Uversky, 2010; Dudzik et al., 2011). Additionally, metals can also perform aberrant interactions with proteins such as beta-amyloid, $\alpha$-synuclein, and prion proteins (Santner and Uversky, 2010; Hong and Simon, 2011). For instance, $\mathrm{Zn}$ and $\mathrm{Al}$ strongly stimulate the aggregation/fibrillogenesis of variations of ataxin-3, a protein involved in the polyglutamine disease ataxia (Ricchelli et al., 2007). In addition, a series of mono, di, and trivalent metal ions such as $\mathrm{Li}, \mathrm{Mn}, \mathrm{Zn}, \mathrm{Cu}, \mathrm{Al}$ can cause significant acceleration of $\alpha$-synuclein fibril formation in vitro (Santner and Uversky, 2010). These processes trigger HSP activation and consequently, a defense response in order to detoxify and protect the cell against the oxidative injury and alterations in the proteostasis caused by the pro-oxidant (Stefani and Dobson, 2003; Dudzik et al., 2011; Hong and Simon, 2011). Several studies using toxicogenomics have demonstrated that HSP levels are elevated following metal exposure in different animal models. In C. elegans, Anbalagan et al. demonstrated that $\mathrm{Cd}, \mathrm{Cu}, \mathrm{Zn}$, and both 
organic $\mathrm{MeHg}$ and inorganic $\mathrm{Hg}$ exposure induced the heat shock genes, HSP-16.1, HSP-16.2, HSP-6, HSP-60 were at least twofold increased, using transgenic GFP strains (Helmcke and Aschner, 2010; Anbalagan et al., 2012). Currently, there is little data unraveling the mechanisms that underlie metal detoxification via HSP, and toxicogenomics will be an important tool in this research.

The ER serves as the major organelle for protein synthesis, modification, folding, and transportation. Disruption of normal ER function results in accumulation of unfolded or misfolded proteins inside ER lumen, known as ER stress, triggering the unfolded protein response (UPR), in order to decrease protein synthesis, enhance protein folding, and degradation. The molecular chaperone GRP78/BiP (immunoglobulin-binding protein) serves as a sensor in this signaling pathway. When ER functions normally, BiP binds to three ER transmembrane proteins, including an endoribonuclease IRE1, protein kinase PERK, and a transcription factor ATF6. Upon ER stress, BiP dissociates from these proteins and binds to unfolded protein to improve folding, thus releasing these three proteins from ER membrane and activating subsequent signaling pathways. These three transmembrane proteins are corresponding to three distinct UPR signaling branches. The transmembrane kinase PERK phosphorylates its downstream target, eukaryotic translation initiation factor (eIF $2 \alpha)$, through which the protein synthesis is shut down. IRE1 cleaves a transcription factor xbp-1 pre-mRNA, which then gets translated and translocated into nucleus to activate genes for protein degradation, such as ER-associated degradation (ERAD) complex. ATF6 dissociates from ER membrane, travels to Golgi apparatus and gets C-terminal cleaved, releasing the $\mathrm{N}$-terminal cytosolic fragment to enter the nucleus, and activate targets genes. These genes are mainly ER resident chaperones (such as $\mathrm{BiP}$ and protein disulfide isomerase), upregulation of which enhances protein folding in the ER. Activation of three UPR branches would alleviate the stress, thus restore ER protein homeostasis; on the other hand, if the stress remains unsolved, apoptosis is activated to kill these cells. To date, ER stress has been found in many human neurological disorder diseases, including early-onset torsion dystonia (Chen et al., 2010), AD (Hoozemans et al., 2005, 2009), PD (Jiang et al., 2010; Calì et al., 2011), amyotrophic lateral sclerosis (Atkin et al., 2008; Nishitoh et al., 2008; Mori et al., 2011), and HD (Reijonen et al., 2008).

Recently heavy metal exposure has been associated with ER stress. Gardarin et al. (2010) found that ER was the major target of $\mathrm{Cd}$ exposure in yeast Saccharomyces cerevisiae. Upon $50 \mu \mathrm{M}$ of Cd treatment, splicing of $H A C 1$ (yeast $x b p-1$ ) mRNA was observed, $\Delta$ ire 1 and $\Delta$ hacl strains showed hypersensitive to $\mathrm{Cd}$, similar to ER stress inducer tunicamycin and dichlorodiphenylchloroethane (DTT; Gardarin et al., 2010). In addition, 14 out of 16 mutants in MAPK signaling pathway, which is important for tolerance of ER stress, is also sensitive to Cd treatment (Gardarin et al., 2010). Fauchon et al. (2002) noticed activation ER chaperones PDI, FKB2, LHS1, and JEM1 and UPR components upon Cd treatment. $\mathrm{MeHg}$ has been shown to induce hermetic changes in GRP78 levels, with low acute doses increasing GRP78 expression but high doses decreased its expression, suggesting that low level MeHg exposure induces cytoprotective ER stress pathway (Zhang et al., 2011). In mammalian cell culture, $\mathrm{Pb}$ (Qian et al., 2001; Shinkai et al., 2010), Mn (Chun et al., 2001), Hg (Qian et al., 2001), Ni (Hiramatsu et al., 2007), Co (Hiramatsu et al., 2007), and Cd (Hiramatsu et al., 2007) also caused ER stress and activated UPR by upregulating $\mathrm{BiP}$ expression. In transgenic mice administered with $\mathrm{Cd}^{2+}$, rapid and transient ER stress was induced in liver and kidney predominantly, but not obvious in other tissues (Hiramatsu et al., 2007). Although it remains unclear whether these heavy metals directly or indirectly interfere with ER functions, restoration of ER homeostasis could be a treatment of these metal exposures. Induction of ER stress has not been reported in C. elegans in response to metal exposure, however there is potential to investigate ER stress in transgenic strains for genes associated with diseases where ER stress has been observed.

\section{METAL-INDUCED APOPTOSIS}

Despite the existence of multiple protective pathways, cellular damage in response to metal exposure may at times be too severe. Exposure to heavy metals has been shown to induce programmed cell death or apoptosis, by influencing various components of several apoptotic pathways (Rana, 2008). However, most of these studies have been in vitro and they do not seem to recapitulate the effects in live animals. Consequently, the C. elegans model system has become a favorable biosensor in studies on the induction of apoptotic pathways in vivo following exposure to various types of toxicants with most studies focusing on germline apoptosis in the nematodes (Wu et al., 2006; Leung et al., 2008). In addition to the physiologically requisite germline apoptosis that occurs in nematodes, stress-induced apoptosis can occur through multiple signaling transduction pathways. Genotoxic insults have been shown to mediate apoptosis through a pathway involving the checkpoint protein HUS-1, the p53 homolog CED-9, and the necessary apoptotic CED-3 caspase (Derry et al., 2001; Hofmann et al., 2002; Conradt and Xue, 2005). However, other stressors can lead to the induction of p53-dependent or independent pathways that require MAPK-related signaling transduction in order for germline apoptosis to occur (Salinas et al., 2006; Rutkowski et al., 2011). The conservation of these pathways in C. elegans allows for the validation on their role in mammalian in vivo metal-induced apoptotic mechanisms (Caffrey et al., 1999).

The C. elegans germline is composed of two U-shaped gonad arms that join proximally at the uterus of the nematode. Both physiologically relevant and stress-induced germline apoptosis occurs at the loop of the gonad (Gartner et al., 2008). A common germline apoptosis assay that can be used in C. elegans involves staining worms with the fluorescent dye acridine orange (AO) that labels the characteristic, highly fragmented DNA of apoptotic cells (Kelly et al., 2000). For example, C. elegans studies found that $\mathrm{Ni}$ exposure can induce germline apoptosis in a dose- and time-dependent manner (Kezhou et al., 2010). This increase in $\mathrm{Ni}$-induced germ cell corpses is independent of the ERK pathway as shown in C. elegans knockout strains for the MAPKKK (lin$45)$, MAPKK ( $m e k-2)$, and MAPK ( $m p k-1)$ homologs. Moreover, mutant worms lacking cep-1 (homolog for the tumor suppressor p53), hus-1, and egl-1 (involved in DNA damage checkpoints) show increased germline apoptosis upon $\mathrm{Ni}$ exposure, and are not required for $\mathrm{Ni}$-induced germline apoptosis when compared to wildtype $\mathrm{Ni}$-exposed worms. However, $\mathrm{Ni}$-induced germline apoptosis is decreased in mutant homologs of the JNK (jkk-1, 
$m e k-1, j n k-1$, and $m k k-4$ loss-of-function strains) and p38 MAPK ( $n s y-1$, sek-1, pmk-1, and pmk-3 loss-of-function strains) signaling cascades, indicating the significance of these two pathways in Ni-induced apoptosis in C. elegans (Kezhou et al., 2010). Similarly, Cd exposure in C. elegans also induces germline apoptosis in a manner dependent upon the JNK and p38 MAPK pathways, as indicated by the blockage of apoptosis in Cd-exposed JNK and p38 MAPK pathway homolog knockouts (except in loss-offunction pmk-3 mutants; Wang et al., 2008). Also analogous to Ni-induced apoptotic pathways, both cep-1 and hus-1 play nonessential roles in germline apoptosis in warms exposed to $50 \mu \mathrm{M}$ Cd (Wang et al., 2008), suggesting the lack of involvement of the DNA damage response in mediating metal-induced apoptosis in C. elegans. However, another gene expression profiling study in C. elegans has found that cep-1 and ape-1 (apoptosis enhancer 1) mRNA is up-regulated upon exposure to $0.85 \mathrm{mg} / \mathrm{L} \mathrm{Cd}$ (Roh et al., 2006). Such a discrepancy illustrates the complex control of these apoptotic pathways that may be highly dose-dependent. Interestingly, a separate study found that cobalt exposure mimicked the same apoptotic pathways delineated in the above studies: DNA damage-independent, but JNK- and p38 MAPK-dependent mechanisms (Chong et al., 2009). These studies indicate consistently shared signaling cascades that mediate apoptosis caused by a variety of metals.

Moreover, metal exposure also mediates apoptosis through increased oxidative stress. For example, $\mathrm{Cu}$ induces its toxicity via ROS generation that damage DNA, lipids, and proteins (Jomova and Valko, 2011). This is thought to via a Fenton-type of reaction (Held et al., 1996). However, the exact pathways involved in $\mathrm{Cu}$-induced apoptosis are not clearly understood. Using C. elegans model, Wang et al. (2009) established that $\mathrm{Cu}$ induces germline apoptosis in analogous fashion to $\mathrm{Ni}$ and $\mathrm{Cd}$. Similar to Ni- and $\mathrm{Cd}$-induced apoptosis, $\mathrm{Cu}$-induced germline apoptosis does not rely on DNA checkpoint genes, as hus-1, clk-1, ced-9, and egl-1 knockouts do not prevent $\mathrm{Cu}$-induced apoptosis. Similar to the other metals, $\mathrm{Cu}$-induced apoptosis does not depend on CEP$1 / \mathrm{p} 53$, as these knockouts caused a significant increase in germline apoptosis upon $\mathrm{Cu}$ exposure. However, knockouts of the caspase ced-3 gene, Apaf-1 homolog ced-4 gene, ERK pathway homologs (lin-45, mek-2, and mpk-1), JNK pathway homologs (nsy-1,3mek$1, j k k-1, m k k-4, j n k-1)$, and some p38 MAPK homologs (sek-1, $p m k-1$, but not $p m k-3$ ) are all essential for $\mathrm{Cu}$-induced germline apoptosis (Wang et al., 2009). The involvement of multiple MAPK signaling cascades suggests that the cell requires more effort to manage the encompassing effects of $\mathrm{Cu}$ toxicity, potentially due to its highly reactive nature in generating ROS in mitochondria to initiate the caspase cascade. However, the DNA damage response remains non-essential in mediating this apoptosis.

\section{APPLICATION OF TOXICOGENOMIC SCREENS IN NEURODEGENERATIVE DISEASES}

Caenorhabditis elegans have been used to investigate several neurological disorders primarily due to its simple and well characterized nervous system. With its short lifespan and ease of genetic mutability, toxicogenomic screens may be quickly and easily performed to identify genes involved in metal-induced neurotoxicity. For example, C. elegans possess only eight dopaminergic (DAergic) neurons, which are easily counted in transgenic worms expressing GFP driven by the dopamine transporter, dat-1, promoter. Dopamine controls specific, measurable behaviors in worms, including food searching behavior, defecation, and egg laying (Sulston et al., 1975; Weinshenker et al., 1995), which are easily measured for loss of DAergic functioning. Herein, we discuss the application of toxigenomic screens in C. elegans to examine the role of metals in PD.

\section{PARKINSON'S DISEASE}

Parkinson's disease is the second most common neurodegenerative disease, afflicting $\sim 2 \%$ of the US population (Bushnell and Martin, 1999). It is characterized by the selective loss of DAergic cells in the substantia nigra pars compacta (SNpc) and locus coeruleus regions of the brain (Wilson et al., 1996). The mechanisms underlying the selective degeneration of the DAergic neurons are poorly understood; however genetic factors and environmental and endogenous toxins have been implicated (Dauer and Przedborski, 2003). Epidemiological studies suggest that PD is more common in rural areas, where the increased prevalence is associated with the use of pesticides, herbicides, and heavy metals (Gorell et al., 2004). Observable symptoms in patients with PD include emotional and cognitive decline, bradykinesia, rigidity, tremors, and postural instability (Lees et al., 2009). In addition to cell loss, proteinaceous intracellular inclusions called Lewy bodies are observed in postmortem brains. The majority of PD cases are sporadic, while $10-20 \%$ of cases have a genetic component. Familial PD may be due to autosomal dominant genes for $\alpha$-synuclein and leucine-rich repeat kinase 2 (LRRK2) or autosomal recessive genes for parkin, DJ-1, and phosphatase and tensin homolog (PTEN)-induced novel kinase 1 (PINK1), all of which have orthologs in worms, such as $p d r-1$ (Parkin), djr-1 (DJ-1), lrk-1 (LRRK2), pink-1 (PINK1), but not $\alpha$-synuclein. Proteinaceous inclusions can be induced in $C$. elegans by overexpression of either wildtype of mutant $\alpha$-synuclein under the dopamine transporter (dat-1) promoter (Kuwahara et al., 2006). Interestingly, mammalian models of $\alpha$-synuclein overexpression have not been successful in causing selective DAergic neurodegeneration, which makes C. elegans an invaluable tool.

In addition to genetic predisposition to $\mathrm{PD}$, environmental exposures contribute to the disease development as well. Exposure to $\mathrm{Mn}$, either from the environment or in occupational settings, can produce Parkinsonian-like symptoms. These symptoms include rigidity, tremor, gait disturbances, and hypokinesia, and are attributed to selective interaction of $\mathrm{Mn}$ with the basal ganglia downstream of the nigrostriatal DAergic projection, which are areas that readily accumulate Mn (Aschner et al., 2007). Mn has been shown oxidize DA to a highly toxic reactive metabolite leukoaminochrome $o$-semiquinone (Graumann et al., 2002). $\mathrm{MeHg}$ exposure has also been implicated in $\mathrm{PD}$. $\mathrm{MeHg}$ poisoning causes severe neurological deficits due to brain lesions and the disruption of neurotransmitter systems (Aschner and Syversen, 2005). Both $\mathrm{PD}$ and $\mathrm{MeHg}$ poisoning present resting tremors and alterations in motor functioning (Biernat et al., 1999; Kaur et al., 2007). MeHg exposure occurs through seafood consumption, due to the global cycling, and accumulation of $\mathrm{Hg}$ from industries that reaches the aquatic environment (Fitzgerald and Clarkson, 1991). 
Several studies have shown an association between PD and fish consumption or occupational exposure to Hg (Biernat et al., 1999; Kirkey et al., 2001; Fabrizio et al., 2007; Petersen et al., 2008a,b). Serum levels of $\mathrm{Hg}$ have been shown to increase post-PD diagnosis, and low serum $\mathrm{Hg}$ levels have been associated with decreased risk for development of PD (Gellein et al., 2008).

Toxicogenomic screens have been performed in C. elegans knockdown strains exposed to $\mathrm{Mn}$ to identify genes that are involved in Mn toxicity. Benedetto et al. (2010) found that Mn exposure induces a dose-dependent degeneration in DAergic neurons in $C$. elegans, which required the presence of the reuptake transporter, DAT-1, as neurodegeneration was not observed in dat-1 knockdown worms. Using various strains of $C$. elegans, Benedetto et al. (2010) also found that toxicity was prevented by the loss of tyrosine hydroxylase (TH)/CAT-2 function in the double knockout strain, cat-2(e1112);dat-1(ok157) and knockdown of vesicular monoamine transporter (VMAT2)/CAT1 in cat-1(e1111) mutants, in which DAergic neurons were unable to release DA at the synaptic cleft. As in mammals, Mn enters DAergic neurons in C. elegans through NRAMP/divalent metal transporters, as deletion of the NRAMP ortholog smf-1 or smf-3 gene attenuated $\mathrm{Mn}$ induced DAergic neurodegeneration and increased survival (Au et al., 2009; Settivari et al., 2009).

The use of C. elegans has also facilitated identification of genes that are involved in the cellular response to Mn exposure. Metals cause oxidative stress through the generation of ROS through Fenton chemistry. Cells respond to increased ROS through upregulation of antioxidant molecules and enzymes via activation of the Nrf2 transcription factor. Benedetto et al. (2010) found that loss-of-function mutants for $s k n-1$, the worm ortholog to Nrf2, had increased sensitivity to Mn toxicity, whereas wildtype worms expressing SKN-1::GFP showed activation of the transcription factor after Mn exposure. Cells can also produce ROS as a defense mechanism using specific enzymatic pathways. Loss-of-function of bli-3 gene, a dual oxidase involved in pathogen-induced ROS production, caused increased resistance to Mn toxicity and surprisingly had no increase in ROS production from Mn exposures, implying that BLI-3 is required for ROS-mediated effects in Mn

\section{REFERENCES}

Al-Shami, S. A., Md Rawi, C. S., Ahmad, A. H., and Mohd Nor, S. A. (2012). Genotoxicity of heavy metals to the larvae of Chironomus kiiensis Tokunaga after short-term exposure. Toxicol. Ind. Health. PMID: 22025505. [Epub ahead of print].

An, J. H., and Blackwell, T. K. (2003). SKN-1 links C. elegans mesendodermal specification to a conserved oxidative stress response. Genes Dev. 17, 1882-1893.

Anbalagan, C., Lafayette, I., AntoniouKourounioti, M., Haque, M., King, J., Johnsen, B., Baillie, D., Gutierrez, C., Rodriguez Martin, J. A., and de Pomerai, D. (2012). Transgenic nematodes as biosensors for metal

exposure (Benedetto et al., 2010). It is unknown if dual oxidases are involved in mammalian responses to $\mathrm{Mn}$.

Although $\mathrm{MeHg}$ exposure causes several toxic effects in $C$. elegans, including decreased survival, developmental delay, and decreased pharyngeal pumping, $\mathrm{MeHg}$ exposure did not cause neurodegeneration (Helmcke et al., 2009; Helmcke and Aschner, 2010). This suggests the presence of protective mechanisms in C. elegans' neurons. $\mathrm{MeHg}$ exposure causes oxidative stress in C. elegans through alterations in GSH levels, increased expression of HSPs, and glutathione-S-transferase (GST; Helmcke et al., 2009; Helmcke and Aschner, 2010). GST levels are controlled by $\mathrm{Nrf}$ 2, which is activated by $\mathrm{MeHg}$ in mammalian glial cells ( $\mathrm{Ni}$ et al., 2010). Knockdown of the $s k n-1$ gene led to DAergic neuron degeneration in $30 \%$ of the worms exposed to $1 \mathrm{mM} \mathrm{MeHg}$, whereas no degeneration was observed in wildtype animals (Vanduyn et al., 2010). Further studies are necessary to understand how C. elegans DAergic neurons are protected from $\mathrm{MeHg}$ induced neurodegeneration.

\section{CONCLUSION}

Many of the pathways and cellular processes affected by metals in humans are also present in C. elegans. This combined with the ease of genetic manipulability and inexpensive cost make toxicogenomic studies in the worm an attractive alternative to mammalian systems. The $C$. elegans model has proved to be an invaluable tool in studying metal-induced gene expression, DNA damage, and apoptosis. The short lifespan has proved useful in performing genetic screens for xenobiotics and the large RNAi libraries available have been utilized to find novel proteins and protein interactions involved in cellular responses to metal exposure. Through the high homology between human and worm genomes novel targets for therapeutic intervention may become apparent through further large scale toxicogenomic studies in the nematode.

\section{ACKNOWLEDGMENTS}

We are grateful for support by NIEHS R01ES07331, R01ES10563, the Center in Molecular Toxicology NIH grant P30ES00267 and the Training Program in Environmental Toxicology grant T32ES007028.

Manganese: recent advances in understanding its transport and neurotoxicity. Toxicol. Appl. Pharmacol. 221, 131-147.

Aschner, M., and Syversen, T. (2005). Methylmercury: recent advances in the understanding of its neurotoxicity. Ther. Drug. Monit. 27, 278-283.

Atkin, J. D., Farg, M. A., Walker, A. K., McLean, C., Tomas, D., and Horne, M. K. (2008). Endoplasmic reticulum stress and induction of the unfolded protein response in human sporadic amyotrophic lateral sclerosis. Neurobiol. Dis. 30, 400-407.

Au, C., Benedetto, A., Anderson, J., Labrousse, A., Erikson, K., Ewbank,
J. J., and Aschner, M. (2009). SMF1, SMF-2 and SMF-3 DMT1 orthologues regulate and are regulated differentially by manganese levels in C. elegans. PLoS ONE 4, e7792. doi:10.1371/journal.pone.0007792

Bal, W., and Kasprzak, K. S. (2002). Induction of oxidative DNA damage by carcinogenic metals. Toxicol. Lett. 127, 55-62.

Benedetto, A., Au, C., Avila, D. S., Milatovic, D., and Aschner, M. (2010). Extracellular dopamine potentiates Mn-induced oxidative stress, lifespan reduction, and dopaminergic neurodegeneration in a BLI-3dependent manner in Caenorhabditis elegans. PLoS Genet. 6, e1001084. doi:10.1371/journal.pgen.1001084 
Beyersmann, D., and Hartwig, A. (2008). Carcinogenic metal compounds: recent insight into molecular and cellular mechanisms. Arch. Toxicol. 82, 493-512.

Biernat, H., Ellias, S. A., Wermuth, L., Cleary, D., de Oliveira Santos, E. C., Jorgensen, P. J., Feldman, R. G., and Grandjean, P. (1999). Tremor frequency patterns in mercury vapor exposure, compared with early Parkinson's disease and essential tremor. Neurotoxicology 20, 945-952.

Bushnell, D. M., and Martin, M. L. (1999). Quality of life and Parkinson's disease: translation and validation of the US Parkinson's Disease Questionnaire (PDQ-39). Qual. Life Res. 8, 345-350.

Caffrey, D. R., O'Neill, L. A., and Shields, D. C. (1999). The evolution of the MAP kinase pathways: coduplication of interacting proteins leads to new signaling cascades. J. Mol. Evol. $49,567-582$

Calì, T., Ottolini, D., and Brini, M. (2011). Mitochondria, calcium, and endoplasmic reticulum stress in Parkinson's disease. Biofactors 37, 228-240.

Chen, P., Burdette, A. J., Porter, J. C., Ricketts, J. C., Fox, S. A., Nery, F. C., Hewett, J. W., Berkowitz, L. A., Breakefield, X. O., Caldwell, K. A., and Caldwell, G. A. (2010). The early-onset torsion dystoniaassociated protein, torsin $\mathrm{A}$, is a homeostatic regulator of endoplasmic reticulum stress response. Hum. Mol. Genet. 19, 3502-3515.

Cheng, R. Y., Zhao, A., Alvord, W. G., Powell, D. A., Bare, R. M., Masuda, A., Takahashi, T., Anderson, L. M., and Kasprzak, K. S. (2003). Gene expression dose-response changes in microarrays after exposure of human peripheral lung epithelial cells to nickel(II). Toxicol. Appl. Pharmacol. 191, 22-39.

Chong, R., Ke-Zhou, C., and ZengLiang, Y. (2009). Induction of germline apoptosis by cobalt and relevant signal transduction pathways in Caenorhabditis elegans. Toxicol. Mech. Methods 19, 541-546.

Chu, K. W., Chan, S. K., and Chow, K. L. (2005). Improvement of heavy metal stress and toxicity assays by coupling a transgenic reporter in a mutant nematode strain. Aquat. Toxicol. 74, 320-332.

Chun, H. S., Lee, H., and Son, J. H. (2001). Manganese induces endoplasmic reticulum (ER) stress and activates multiple caspases in nigral dopaminergic neuronal cells, SN4741. Neurosci. Lett. 316, 5-8.
Conradt, B., and Xue, D. (2005). "Programmed cell death," in WormBook, ed. The C. elegans Research Community, 1-13.

Cui, Y., McBride, S. J., Boyd, W. A., Alper, S., and Freedman, J. H. (2007). Toxicogenomic analysis of Caenorhabditis elegans reveals novel genes and pathways involved in the resistance to cadmium toxicity. Genome Biol. 8, R122.

Dauer, W., and Przedborski, S. (2003). Parkinson's disease: mechanisms and models. Neuron 39, 889-909.

Denver, D. R., Dolan, P. C., Wilhelm, L. J., Sung, W., Lucas-Lledo, J. I., Howe, D. K., Lewis, S. C., Okamoto, K., Thomas, W. K., Lynch, M., and Baer, C. F. (2009). A genome-wide view of Caenorhabditis elegans base-substitution mutation processes. Proc. Natl. Acad. Sci. U.S.A. 106, 16310-16314.

Derry, W. B., Putzke, A. P., and Rothman, J. H. (2001). Caenorhabditis elegans p53: role in apoptosis, meiosis, and stress resistance. Science 294, 591-595.

Dudzik, C. G., Walter, E. D., and Millhauser, G. L. (2011). Coordination features and affinity of the $\mathrm{Cu}(2)+$ site in the alpha-synuclein protein of Parkinson's disease. Biochemistry 50, 1771-1777.

Escobar Mdel, C., Souza, V., Bucio, L., Hernandez, E., Gomez-Quiroz, L. E., and Gutierrez Ruiz, M. C. (2009). MAPK activation is involved in cadmium-induced Hsp70 expression in HepG2 cells. Toxicol. Mech. Methods 19, 503-509.

Fabrizio, E., Vanacore, N., Valente, M., Rubino, A., and Meco, G. (2007). High prevalence of extrapyramidal signs and symptoms in a group of Italian dental technicians. BMC Neurol. 7, 24. doi:10.1186/1471-2377-7-24

Fauchon, M., Lagniel, G., Aude, J.-C., Lombardia, L., Soularue, P., Petat, C., Marguerie, G., Sentenac, A., Werner, M., and Labarre, J. (2002). Sulfur sparing in the yeast proteome in response to sulfur demand. Mol. Cell 9, 713-723.

Fitzgerald, W. F., and Clarkson, T. W. (1991). Mercury and monomethylmercury: present and future concerns. Environ. Health Perspect. 96, 159-166.

Frydman, J. (2001). Folding of newly translated proteins in vivo: the role of molecular chaperones. Annu. Rev. Biochem. 70, 603-647.

Fujii, M., Adachi, N., Shikatani, K., and Ayusawa, D. (2009). [FeFe]hydrogenase-like gene is involved in the regulation of sensitivity to oxygen in yeast and nematode. Genes Cells 14, 457-468.

Gardarin, A., Chédin, S., Lagniel, G., Aude, J.-C., Godat, E., Catty, P., and Labarre, J. (2010). Endoplasmic reticulum is a major target of cadmium toxicity in yeast. Mol. Microbiol. 76, 1034-1048.

Gartner, A., Boag, P. R., and Blackwell, T. K. (2008). Germline survival and apoptosis. WormBook 1-20.

Gellein, K., Syversen, T., Steinnes, E., Nilsen, T. I., Dahl, O. P., Mitrovic, S., Duraj, D., and Flaten, T. P. (2008). Trace elements in serum from patients with Parkinson's disease - a prospective case-control study: the Nord-Trondelag Health Study (HUNT). Brain Res. 1219, 111-115.

Gidalevitz, T., Ben-Zvi, A., Ho, K. H. Brignull, H. R., and Morimoto, R. I. (2006). Progressive disruption of cellular protein folding in models of polyglutamine diseases. Science 311, 1471-1474.

Gidalevitz, T., Krupinski, T., Garcia, S., and Morimoto, R. I. (2009). Destabilizing protein polymorphisms in the genetic background direct phenotypic expression of mutant SOD1 toxicity. PLoS Genet. 5, e1000399. doi:10.1371/journal.pgen.1000399

Gitler, A. D., Chesi, A., Geddie, M. L., Strathearn, K. E., Hamamichi, S., Hill, K. J., Caldwell, K. A., Caldwell, G. A., Cooper, A. A., Rochet, J. C., and Lindquist, S. (2009). Alpha-synuclein is part of a diverse and highly conserved interaction network that includes PARK9 and manganese toxicity. Nat. Genet. 41, 308-315.

Gorell, J. M., Peterson, E. L., Rybicki, B. A., and Johnson, C. C. (2004) Multiple risk factors for Parkinson's disease. J. Neurol. Sci. 217, 169-174.

Graumann, R., Paris, I., MartinezAlvarado, P., Rumanque, P., PerezPastene, C., Cardenas, S. P., Marin, P., Diaz-Grez, F., Caviedes, R. Caviedes, P., and Segura-Aguilar, J. (2002). Oxidation of dopamine to aminochrome as a mechanism for neurodegeneration of dopaminergic systems in Parkinson's disease. Possible neuroprotective role of DTdiaphorase. Pol. J. Pharmacol. 54, 573-579.

Greer, E. L., Maures, T. J., Ucar, D., Hauswirth, A. G., Mancini, E., Lim, J. P., Benayoun, B. A., Shi, Y., and Brunet,A. (2011). Transgenerational epigenetic inheritance of longevity in Caenorhabditis elegans. Nature $479,365-371$.
Hall, S. E., Beverly, M., Russ, C., Nusbaum, C., and Sengupta, P. (2010). A cellular memory of developmental history generates phenotypic diversity in C. elegans. Curr. Biol. 20, 149-155.

Hartman, P. S., Ishii, N., Kayser, E. B., Morgan, P. G., and Sedensky, M. M. (2001). Mitochondrial mutations differentially affect aging, mutability and anesthetic sensitivity in Caenorhabditis elegans. Mech. Ageing Dev. 122, 1187-1201.

Held, K. D., Sylvester, F. C., Hopcia, K. L., and Biaglow, J. E. (1996). Role of Fenton chemistry in thiol-induced toxicity and apoptosis. Radiat. Res. 145, 542-553.

Helmcke, K. J., and Aschner, M. (2010). Hormetic effect of methylmercury on Caenorhabditis elegans. Toxicol. Appl. Pharmacol. 248, 156-164.

Helmcke, K. J., Syversen, T., Miller, D. M. III, and Aschner, M. (2009). Characterization of the effects of methylmercury on Caenorhabditis elegans. Toxicol. Appl. Pharmacol. 240, 265-272.

Hiramatsu, N., Kasai, A., Du, S., Takeda, M., Hayakawa, K., Okamura, M. Yao, J., and Kitamura, M. (2007). Rapid, transient induction of ER stress in the liver and kidney after acute exposure to heavy metal: evidence from transgenic sensor mice. FEBS Lett. 581, 2055-2059.

Hofmann, E. R., Milstein, S., Boulton, S. J., Ye, M., Hofmann, J. J., Stergiou, L., Gartner, A., Vidal, M., and Hengartner, M. O. (2002). Caenorhabditis elegans HUS-1 is a DNA damage checkpoint protein required for genome stability and EGL-1mediated apoptosis. Curr. Biol. 12, 1908-1918.

Honda, Y., and Honda, S. (1999). The daf-2 gene network for longevity regulates oxidative stress resistance and $\mathrm{Mn}$-superoxide dismutase gene expression in Caenorhabditis elegans. FASEB J. 13, 1385-1393.

Hong, L., and Simon, J. D. (2011). Insights into the thermodynamics of copper association with amyloidbeta, alpha-synuclein and prion proteins. Metallomics 3, 262-266.

Hoozemans, J. J. M., van Haastert, E. S., Nijholt, D. A. T., Rozemuller, A. J. M., Eikelenboom, P., and Scheper W. (2009). The unfolded protein response is activated in pretangle neurons in Alzheimer's disease hippocampus. Am. J. Pathol. 174, 1241-1251.

Hoozemans, J. J. M., Veerhuis, R., Van Haastert, E. S., Rozemuller, J. M., Baas, F., Eikelenboom, P., and Scheper, W. (2005). The unfolded 
protein response is activated in Alzheimer's disease. Acta Neuropathol. 110, 165-172.

Imaizumi, K., Miyoshi, K., Katayama, T., Yoneda, T., Taniguchi, M., Kudo, T., and Tohyama, M. (2001). The unfolded protein response and Alzheimer's disease. Biochim. Biophys. Acta 1536, 85-96.

Ishii, N., Takahashi, K., Tomita, S., Keino, T., Honda, S., Yoshino, K., and Suzuki, K. (1990). A methyl viologen-sensitive mutant of the nematode Caenorhabditis elegans. Mutat. Res. 237, 165-171.

Jiang, P., Gan, M., Ebrahim, A., Lin, W.-L., Melrose, H., and Yen, S.-H. (2010). ER stress response plays an important role in aggregation of alpha-synuclein. Mol. Neurodegener. 5,56 .

Jomova, K., and Valko, M. (2011). Advances in metal-induced oxidative stress and human disease. Toxicology 283, 65-87.

Kaletta, T., and Hengartner, M. O. (2006). Finding function in novel targets: C. elegans as a model organism. Nat. Rev. Drug Discov. 5, 387-398.

Kaur, P., Aschner, M., and Syversen, T. (2007). Role of glutathione in determining the differential sensitivity between the cortical and cerebellar regions towards mercuryinduced oxidative stress. Toxicology 230, 164-177.

Kayser, E. B., Morgan, P. G., Hoppel, C. L., and Sedensky, M. M. (2001). Mitochondrial expression and function of GAS-1 in Caenorhabditis elegans. J. Biol. Chem. 276, 20551-20558.

Kelly, K. O., Dernburg, A. F., Stanfield, G. M., and Villeneuve, A. M. (2000). Caenorhabditis elegans msh5 is required for both normal and radiation-induced meiotic crossing over but not for completion of meiosis. Genetics 156, 617-630.

Kezhou, C., Chong, R., and Zengliang, Y. (2010). Nickel-induced apoptosis and relevant signal transduction pathways in Caenorhabditis elegans. Toxicol. Ind. Health 26, 249-256.

Kirkey, K. L., Johnson, C. C., Rybicki, B. A., Peterson, E. L., Kortsha, G. X., and Gorell, J. M. (2001). Occupational categories at risk for Parkinson's disease. Am. J. Ind. Med. 39, 564-571.

Kordas, K. (2010). Iron, lead, and children's behavior and cognition. Annu. Rev. Nutr. 30, 123-148.

Koyashiki, G. A., Paoliello, M. M., and Tchounwou, P. B. (2010). Lead levels in human milk and children's health risk: a systematic review. Rev. Environ. Health 25, 243-253.

Kuwahara, T., Koyama, A., GengyoAndo, K., Masuda, M., Kowa, H., Tsunoda, M., Mitani, S., and Iwatsubo, T. (2006). Familial Parkinson mutant alpha-synuclein causes dopamine neuron dysfunction in transgenic Caenorhabditis elegans. J. Biol. Chem. 281, 334-340.

Lees, A. J., Hardy, J., and Revesz, T. (2009). Parkinson's disease. Lancet 373, 2055-2066.

Leung, M. C., Williams, P. L., Benedetto, A., Au, C., Helmcke, K. J., Aschner, M., and Meyer, J. N. (2008). Caenorhabditis elegans: an emerging model in biomedical and environmental toxicology. Toxicol. Sci. 106, 5-28.

McDonald, P. W., Jessen, T., Field, J. R., and Blakely, R. D. (2006). Dopamine signaling architecture in Caenorhabditis elegans. Cell. Mol. Neurobiol. 26, 593-618.

Mori, A., Yamashita, S., Uchino, K., Suga, T., Ikeda, T., Takamatsu, K., Ishizaki, M., Koide, T., Kimura, E., Mita, S., Maeda, Y., Hirano, T., and Uchino, M. (2011). Derlin-1 overexpression ameliorates mutant SOD1-induced endoplasmic reticulum stress by reducing mutant SOD1 accumulation. Neurochem. Int. 58, 344-353.

Neal, A. P., and Guilarte, T. R. (2010). Molecular neurobiology of lead $(\mathrm{Pb}(2+))$ : effects on synaptic function. Mol. Neurobiol. 42, 151-160.

Ni, M., Li, X., Yin, Z., Jiang, H., SidorykWegrzynowicz, M., Milatovic, D., Cai, J., and Aschner, M. (2010). Methylmercury induces acute oxidative stress, altering Nrf2 protein level in primary microglial cells. Toxicol. Sci. 116, 590-603.

Nishitoh, H., Kadowaki, H., Nagai, A., Maruyama, T., Yokota, T., Fukutomi, H., Noguchi, T., Matsuzawa, A., Takeda, K., and Ichijo, H. (2008). ALS-linked mutant SOD1 induces ER stress- and ASK1-dependent motor neuron death by targeting Derlin-1. Genes Dev. 22, 1451-1464.

Petersen, M. S., Halling, J., Bech, S., Wermuth, L., Weihe, P., Nielsen, F., Jorgensen, P. J., Budtz-Jorgensen, E., and Grandjean, P. (2008a). Impact of dietary exposure to food contaminants on the risk of Parkinson's disease. Neurotoxicology 29, 584-590.

Petersen, M. S., Weihe, P., Choi, A., and Grandjean, P. (2008b). Increased prenatal exposure to methylmercury does not affect the risk of Parkinson's disease. Neurotoxicology 29, 591-595.
Powers, M. V., Jones, K., Barillari, C., Westwood, I., van Montfort, R. L., and Workman, P. (2010). Targeting HSP70: the second potentially druggable heat shock protein and molecular chaperone? Cell Cycle 9, 1542-1550.

Qian, Y., Falahatpisheh, M., Zheng, Y., Ramos, K., and Tiffany-Castiglioni, E. (2001). Induction of $78 \mathrm{kD}$ glucose-regulated protein (GRP78) expression and redox-regulated transcription factor activity by lead and mercury in C6 rat glioma cells. Neurotox. Res. 3, 581-589.

Qian, Y., Zheng, Y., Ramos, K. S. and Tiffany-Castiglioni, E. (2005). GRP78 compartmentalized redistribution in Pb-treated glia: role of GRP78 in lead-induced oxidative stress. Neurotoxicology 26, 267-275.

Rana, S. V. (2008). Metals and apoptosis: recent developments. J. Trace Elem. Med. Biol. 22, 262-284.

Reijonen, S., Putkonen, N., Nørremølle, A., Lindholm, D., and Korhonen, L. (2008). Inhibition of endoplasmic reticulum stress counteracts neuronal cell death and protein aggregation caused by N-terminal mutant huntingtin proteins. Exp. Cell Res. 314, 950-960

Ricchelli, F., Fusi, P., Tortora, P., Valtorta, M., Riva, M., Tognon, G., Chieregato, K., Bolognin, S., and Zatta, P. (2007). Destabilization of non-pathological variants of ataxin3 by metal ions results in aggregation/fibrillogenesis. Int. J. Biochem. Cell Biol. 39, 966-977.

Robinson, J. F., Port, J. A., Yu, X., and Faustman, E. M. (2010). Integrating genetic and toxicogenomic information for determining underlying susceptibility to developmental disorders. Birth Defects Res. Part A Clin. Mol. Teratol. 88, 920-930.

Rodella, L. F., Ricci, F., Borsani, E. Stacchiotti, A., Foglio, E., Favero, G. Rezzani, R., Mariani, C., and Bianchi, R. (2008). Aluminium exposure induces Alzheimer's disease-like histopathological alterations in mouse brain. Histol. Histopathol. 23, 433-439.

Roh, J. Y., Lee, J., and Choi, J. (2006) Assessment of stress-related gene expression in the heavy metalexposed nematode Caenorhabditis elegans: a potential biomarker for metal-induced toxicity monitoring and environmental risk assessment. Environ. Toxicol. Chem. 25, 2946-2956.

Roh, J. Y., Sim, S. J., Yi, J., Park, K., Chung, K. H., Ryu, D. Y., and Choi, J. (2009). Ecotoxicity of silver nanoparticles on the soil nematode Caenorhabditis elegans using functional ecotoxicogenomics. Environ. Sci. Technol. 43, 3933-3940.

Rutkowski, R., Dickinson, R., Stewart, G., Craig, A., Schimpl, M., Keyse, S. M., and Gartner, A. (2011). Regulation of Caenorhabditis elegans p53/CEP-1-dependent germ cell apoptosis by Ras/MAPK signaling. PLoS Genet. 7, e1002238. doi:10.1371/journal.pgen.1002238

Salinas, L. S., Maldonado, E., and Navarro, R. E. (2006). Stressinduced germ cell apoptosis by a $\mathrm{p} 53$ independent pathway in Caenorhabditis elegans. Cell Death Differ. 13, 2129-2139.

Salnikow, K., and Zhitkovich, A. (2008). Genetic and epigenetic mechanisms in metal carcinogenesis and cocarcinogenesis: nickel, arsenic, and chromium. Chem. Res. Toxicol. 21, 28-44.

Samuelson, A. V., Carr, C. E., and Ruvkun, G. (2007). Gene activities that mediate increased life span of C. elegans insulin-like signaling mutants. Genes Dev. 21, 2976-2994.

Santner, A., and Uversky, V. N. (2010). Metalloproteomics and metal toxicology of alpha-synuclein. Metallomics 2, 378-392.

Settivari, R., Levora, J., and Nass, R. (2009). The divalent metal transporter homologues SMF-1/2 mediate dopamine neuron sensitivity in Caenorhabditis elegans models of manganism and Parkinson disease. J. Biol. Chem. 284, 35758-35768.

Sharma, S. K., Goloubinoff, P., and Christen, P. (2008). Heavy metal ions are potent inhibitors of protein folding. Biochem. Biophys. Res. Commun. 372, 341-345.

Shinkai, Y., Yamamoto, C., and Kaji, T. (2010). Lead Induces the expression of endoplasmic reticulum chaperones GRP78 and GRP94 in vascular endothelial cells via the JNK-AP-1 pathway. Toxicol. Sci. 114, 378-386.

Sobkowiak, R., and Lesicki, A. (2009). Genotoxicity of nicotine in cell culture of Caenorhabditis elegans evaluated by the comet assay. Drug Chem. Toxicol. 32, 252-257.

Soti, C., Nagy, E., Giricz, Z., Vigh, L., Csermely, P., and Ferdinandy, P. (2005). Heat shock proteins as emerging therapeutic targets. $\mathrm{Br}$. $\mathrm{J}$. Pharmacol. 146, 769-780.

Squitti, R. (2012). Metals in Alzheimer's disease: a systemic perspective. Front. Biosci. 17, 451-472.

Stefani, M., and Dobson, C. M. (2003). Protein aggregation and aggregate toxicity: new insights into protein folding, misfolding diseases and 
biological evolution. J. Mol. Med. 81, 678-699.

Sulston, J., Dew, M., and Brenner, S. (1975). Dopaminergic neurons in the nematode Caenorhabditis elegans. J. Comp. Neurol. 163, 215-226.

Sulston, J. E., and Horvitz, H. R. (1977). Post-embryonic cell lineages of the nematode, Caenorhabditis elegans. Dev. Biol. 56, 110-156.

Vanduyn, N., Settivari, R., Wong, G., and Nass, R. (2010). SKN-1/Nrf2 inhibits dopamine neuron degeneration in a Caenorhabditis elegans model of methylmercury toxicity. Toxicol. Sci. 118, 613-624.

Wang, S., Tang, M., Pei, B., Xiao, X., Wang, J., Hang, H., and $\mathrm{Wu}$, L. (2008). Cadmium-induced germline apoptosis in Caenorhabditis elegans: the roles of HUS1, p53, and MAPK signaling pathways. Toxicol. Sci. 102, 345-351.

Wang, S., Wu, L., Wang, Y., Luo, X., and Lu, Y. (2009). Copper-induced germline apoptosis in Caenorhabditis elegans: the independent roles of DNA damage response signaling and the dependent roles of MAPK cascades. Chem. Biol. Interact. 180, 151-157.

Weinshenker, D., Garriga, G., and Thomas, J. H. (1995). Genetic and pharmacological analysis of neurotransmitters controlling egg laying in C. elegans. J. Neurosci. 15, 6975-6985.

Wenzel, D., Palladino, F., and JedrusikBode, M. (2011). Epigenetics in C. elegans: facts and challenges. Genesis $49,647-661$.

Wilson, J. M., Levey, A. I., Rajput, A., Ang, L., Guttman, M., Shannak, K., Niznik, H. B., Hornykiewicz, O., Pifl, C., and Kish, S. J. (1996). Differential changes in neurochemical markers of striatal dopamine nerve terminals in idiopathic Parkinson's disease. Neurology 47, 718-726.
Wu, Y., Wu, Z., Butko, P., Christen, Y., Lambert, M. P., Klein, W. L., Link, C. D., and Luo, Y. (2006). Amyloidbeta-induced pathological behaviors are suppressed by Ginkgo biloba extract EGb 761 and ginkgolides in transgenic Caenorhabditis elegans. J. Neurosci. 26, 13102-13113.

Yamamoto, K., Honda, S., and Ishii, N. (1996). Properties of an oxygensensitive mutant mev- 3 of the nematode Caenorhabditis elegans. Mutat. Res. 358, 1-6.

Zhang, Y., Yu, F., Lu, R., Liu, Y., Wu, Y., Qian, H., Zhao, X., Wang, S., Xing, G., and Aschner, $M$. (2011). Hormetic effects of acute methylmercury exposure on GRP78 expression in rat brain cortex. DoseResponse. (in press).

Conflict of Interest Statement: The authors declare that the research was conducted in the absence of any commercial or financial relationships that could be construed as a potential conflict of interest.

Received: 25 January 2012; paper pending published: 23 February 2012; accepted: 20 March 2012; published online: 10 April 2012.

Citation: Caito S, Fretham S, MartinezFinley E, Chakraborty S, Avila D, Chen $P$ and Aschner $M$ (2012) Genomewide analyses of metal responsive genes in Caenorhabditis elegans. Front. Gene. 3:52. doi: 10.3389/fgene.2012.00052

This article was submitted to Frontiers in Toxicogenomics, a specialty of Frontiers in Genetics.

Copyright (C) 2012 Caito, Fretham, Martinez-Finley, Chakraborty, Avila, Chen and Aschner. This is an open-access article distributed under the terms of the Creative Commons Attribution Non Commercial License, which permits noncommercial use, distribution, and reproduction in other forums, provided the original authors and source are credited. 